\title{
Semi-random mutagenesis profile of BCR-ABL during imatinib resistance acquirement in $\mathrm{K562}$ cells
}

\author{
YAN DONG $^{1}$, XIAOTONG GAO ${ }^{1}$, YINGXIN ZHAO ${ }^{1}$, \\ MENGYING WEI ${ }^{2}$, LINGMIN XU ${ }^{1}$, GUODONG YANG ${ }^{2}$ and LI LIU ${ }^{1}$ \\ ${ }^{1}$ Department of Hematology, Tangdu Hospital, Fourth Military Medical University, Xi'an, Shaanxi 710038; \\ ${ }^{2}$ Department of Biochemistry and Molecular Biology, Fourth Military Medical University, Xi'an, Shaanxi 710032, P.R. China
}

Received May 9, 2017; Accepted September 1, 2017

DOI: $10.3892 / \mathrm{mmr} .2017 .7835$

\begin{abstract}
Although imatinib is effective in chronic myeloid leukemia treatment, imatinib resistance due to the T315I mutation and/or other mutations is a challenge to be overcome. However, how DNA mutation occurs, particularly the T315I mutation, remains unclear. In the current study, the mutagenesis of BCR-ABL was analyzed via focusing on the process of drug resistance, rather than the final results. Clone sequencing of the BCR-ABL gene and other control genes was applied in two imatinib-resistant cell models. The results have indicated that imatinib actively and selectively causes sporadic mutations in the BCR-ABL gene, however not in the control genes. The majority of the mutations of BCR-ABL were not the clinically observed T315I mutation, suggesting that the T315I mutation may be due to clonal expansion of cells with survival advantages. Taken together, the results of the current study elucidated the mutagenesis process during drug resistance and thus aids in the management of chemotherapy.
\end{abstract}

\section{Introduction}

The annual incidence of newly diagnosed chronic myeloid leukemia (CML) in the United States is estimated to be 4,800 5,200 (1). CML is characterized by the generation of the Philadelphia chromosome, a direct result of the $\mathrm{t}(9 ; 22)$ (q34; q11) balanced reciprocal translocation. This chromosomal translocation leads to expression of fused BCR-ABL, which is an oncogenic fusion protein

Correspondence to: Dr Guodong Yang, Department of Biochemistry and Molecular Biology, Fourth Military Medical University, 169 Changlexi Road, Xi'an, Shaanxi 710032, P.R. China E-mail: yanggd@fmmu.edu.cn

Dr Li Liu, Department of Hematology, Tangdu Hospital, Fourth Military Medical University, 569 Xinsi Road, Xi'an, Shaanxi 710038, P.R. China

E-mail: liuli1@medmail.com.cn

Key words: imatinib resistance, chronic myeloid leukemia, BCR-ABL, gene mutation with constitutive ABL tyrosine kinase activity. BCR-ABL can transform myeloid progenitor cells and drives the development of CML in $95 \%$ cases (2).

Imatinib mesylate (IM), the first-line treatment for CML, is a tyrosine kinase inhibitor (TKI), which binds to the ABL kinase domain and blocks the kinase activity of BCR-ABL, thus inhibiting phosphorylation of substrates (3). IM has been proven to be highly effective, as approximately $80 \%$ of patients in the chronic phase achieve a complete cytogenetic remission within 12 months of therapy (4).

However, approximately $15-20 \%$ of patients ultimately develop resistance to imatinib, which then progresses to an accelerated phase and eventually to a blast crisis (5). The most common mechanism responsible for imatinib resistance are point mutations within the ABL1 kinase domain of BCR-ABL1, which either directly interferes with imatinib binding at critical contact points or prevents the BCR-ABL1 molecule from assuming the appropriate conformation that allows imatinib to bind (4). The T315I mutation, one of the most common mutations of BCR-ABL, occurs when threonine at amino acid position 315 (in the ABL sequence) is replaced with isoleucine, which is responsible for $20 \%$ of imatinib-resistant cases $(6,7)$. Once mutated, T315I is unable to be completely eradicated by the rational combination of TKIs (8). However, how DNA mutation occurs, in particular the T315I mutation, remains unclear. Specifically, whether ABL1 is preferentially mutated or randomly mutated upon imatinib treatment when compared with other genes remains unknown. In addition, whether the T315I mutation and/or other mutations endowing imatinib resistance are specifically induced by imatinib or randomly induced but selectively chosen by imatinib remains unclear. Elucidation of the detailed mechanism would aid in the management of imatinib resistance.

In the present study, the mutagenesis of BCR-ABL was analyzed via focusing on the process of drug resistance, rather than the final results. Clone sequencing was used to study the BCR-ABL gene and other control genes in two imatinib resistant cell models. The results indicated that imatinib actively and selectively causes random sporadic mutations of BCR-ABL over other genes in the genome, while the clinically observed T315I mutation may be due to clonal expansion of cells with a survival advantage. 


\section{Materials and methods}

Cell culture. The K562 and K562G cell lines were originally purchased from the American Type Culture Collection (Manassas, VA, USA). Cells were cultured in RPMI-1640 medium (Hyclone, Logan, UT, USA) supplemented with $10 \%$ fetal bovine serum at $37^{\circ} \mathrm{C}$ containing $5 \% \mathrm{CO}_{2} . \mathrm{K} 562 \mathrm{G}$ cells were originally induced with $0.5-1.0 \mu \mathrm{M}$ imatinib and cultivated over 10 passages. The cells were passaged every other day.

Induction of imatinib resistance. Imatinib-resistant K562 cells (K562R) were developed by exposures of K562 cells to a concentration of $10 \mathrm{nM}$ imatinib. Cells were grown for 10 days. Resistant cells were washed with RPMI-1640 medium and were maintained in RPMI-1640 medium supplemented with 10\% FBS (Excell Bio, Shanghai, China) and $10 \mathrm{nM}$ imatinib.

Cell Counting kit-8 (CCK-8) analysis of cell survival. CCK-8 was used to measure cell viability. Exponentially growing K562 cells, K562R cells and K562G cells were seeded into 96-well plates at density of 2,000 cells per $100 \mu 1$, respectively. Cells were treated with or without $1 \mu \mathrm{M}$ imatinib. Cells with the above treatments were additionally cultured for $12,24,48$, 72,96 and $120 \mathrm{~h}$. All the experiments were performed in five replicates. A total of $2 \mathrm{~h}$ prior to measuring the absorbance, $10 \mu$ l CCK-8 solution (Dojindo Molecular Technologies, Inc., Tokyo, Japan) was added to each well. The absorbance values (optical density) was measured at the wavelength of $450 \mathrm{~nm}$ in each well.

Apoptosis analysis. Apoptosis was determined by 2-color flow cytometry with Annexin V (5 $\mu \mathrm{l} / \mathrm{sample}$; BD Pharmingen, San Diego, CA, USA) and 7-amino-actinomycin D (7-AAD; $10 \mu \mathrm{l} / \mathrm{sample}$ ) staining using $5 \times 10^{5}$ cells per sample.

Mutation analysis. Total RNA was isolated from the cells with different treatments using the TriPure reagent (Roche Diagnostics GmbH, Mannheim, Germany). The first-strand cDNA synthesis reaction from total RNA was catalyzed with Superscript III reverse transcriptase (Invitrogen; Thermo Fisher Scientific, Inc., Waltham, MA, USA) using random primers (hexamers; Invitrogen; Thermo Fisher Scientific, Inc.). For polymerase chain reaction (PCR) cloning, specific oligonucleotide primers were used as follows: ABL, forward, 5'-GACATCACCATGAAGCACAAGC-3' and reverse 5'-CAG CTCCTTTTCCACTTCGTC-3'; ACTB, forward 5'-GTTGCT ATCCAGGCTGTGCTATCC-3' and reverse 5'-AGAAGAGCT ACGAGCTGCCTGACG-3'. The primers were designed based on the gene sequences, respectively. The amplified fragments were cloned into $\mathrm{T}$ vector pMD19 (Takara Bio, Inc., Otsu, Japan) and sequenced by Sunny Biotech (Shanghai Sunny Biotech Co., Ltd., Shanghai, China). Sequence analysis and comparison was conducted using the Basic Local Alignment Search Tool from NCBI (https://blast.ncbi.nlm.nih.gov/Blast.cgi).

Statistical analysis. Results are expressed as the mean \pm standard deviation for measurement data and were analyzed by student's t-test for two group comparison or one-way analysis of variance with Tukey's post hoc test for multiple group analysis. The enumeration data comparison was completed using the Chi-square test. $\mathrm{P}<0.05$ was considered to indicate a statistically significant difference.

\section{Results}

Construction of imatinib-resistant K562 cell models. K562G cells, which were originally induced with $0.5-1.0 \mu \mathrm{M}$ imatinib, were cultivated over 10 passages and included as a control. To identify the early effects of imatinib treatment on BCR-ABL gene mutation, K562 cells were exposed to $10 \mathrm{nM}$ imatinib for 10 days (Fig. 1). Cell Counting Kit-8 and apoptosis analysis were used to compare imatinib resistance in parental K562 cells, K562R cells and K562G cells. The surviving cells were significantly decreased in K562 cells following $24 \mathrm{~h}$ of $1 \mu \mathrm{M}$ imatinib treatment, while no significant reduction in the cell survival rate was observed in K562R and K562G cells (Fig. 2A). Accordingly, there was significant and clear apoptosis observed in K562 cells, however not in K562R and K562G cells (Fig. 2B and C). Notably there were $~ 10 \%$ apoptotic and necrotic cells in the group without imatinib, which should be attributed to the alcohol fixation for flow cytometry analysis, which was comparable among groups. All of these data suggest that both K562R and K562G represent two types of imatinib-resistant cells.

Preferential point mutation in BCR-ABL gene in imatinib resistant cells. Due to the fact that DNA mutation serves an essential role in imatinib resistance in CML, the BCR-ABL and control gene mutations in K562 cells, K562R and K562G cells were investigated. The RNA was isolated and reverse transcribed for PCR amplification of ABL and control genes. The amplicon was cloned into pMD19T and the clones were randomly selected for sequencing to determine the detailed DNA mutation (Fig. 1). All of the selected clones of ABL genes in K562 were not mutated (Table I). In contrast, 4/11 clones from K562G exhibited ABL gene mutations. Two of them were silent mutations (causing no change of the amino acid sequence), and the others were missense mutations (Table I, Fig. 3). Due to the fact there were no silent mutations observed in the control K562 cells, it is thus impossible that the silent mutations origin from the two copies of the parental alleles. Notably, a frameshift mutation and a silent mutation occurred in the same clone of K562G (Table I). For K562R, 6/9 clones displayed ABL point mutations, including 5 silent mutations and 4 missense mutations (Table I, Fig. 3). Significant differences were observed in BCR-ABL1 mutations between the control and K562R cells. There was also a significant difference of the mutation rate between BCR-ABL1 and control gene in K562R cells. Notably, there were no significant differences identified in $\mathrm{K} 562 \mathrm{G}$ cells when compared with the control K562 cell or the control gene, which may be due to the sample size. Increasing the clone numbers detected would increase the reliability of the data.

Rare point mutation in control genes occurs in imatinib resistant cells. In order to determine whether imatinib-resistant cells selectively exhibited gene mutations in the ABL gene, the mutation rates between $\mathrm{ABL}$ and control genes, such as ACTB, were investigated. As presented in Table I, there were 

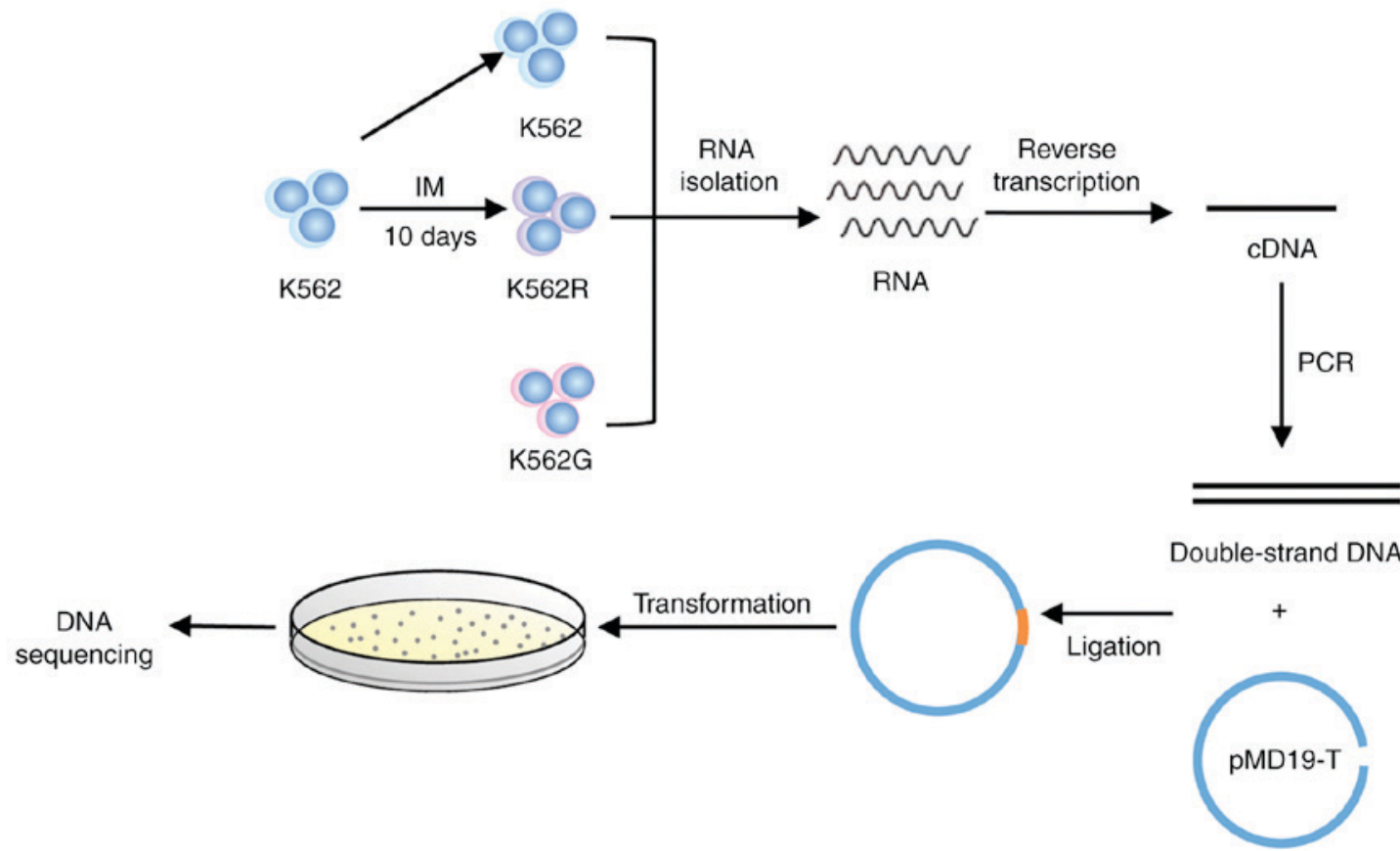

Figure 1. Schematic representation of the experimental procedure. Two imatinib resistant K562 cells, K562R and K562G were included in the study. K562R was developed by selection of the survived K562 cells following exposure of $10 \mathrm{nM}$ imatinib for 10 days and $\mathrm{K} 562 \mathrm{G}$ was originally induced with $0.5-1.0 \mu \mathrm{M}$ imatinib and cultivated over 10 passages. The RNAs of K562 cells and the above two imatinib resistant cell models were isolated and reverse transcribed for PCR amplification of ABL and control genes. The amplified fragments were cloned into T vector pMD19, and the clones were randomly selected for sequencing the detailed DNA mutation. PCR, polymerase chain reaction; IM, imatinib mesylate.

no mutated clones of both ABL and the ACTB control gene present in K562 cells. In imatinib-resistant cells (K562G and $\mathrm{K} 562 \mathrm{R}$ ), only one clone had a mutation in ACTB, which was reduced compared with that of the ABL gene mutation. In addition, the mutation rate of 5S RNA was measured, which yielded similar results as ACTB (data not shown).

Proposed hypothesis of selective BCR-ABL mutation in imatinib resistant cells. Previously, RNA editing and transcription-associated DNA damage have been identified to be responsible for the observed DNA mutations (9-11). It is thus highly possible that blockade of BCR-ABL activity by imatinib treatment would cause the compensatory transcriptional increase of BCR-ABL, which would increase the chance of naked BCR-ABL gene exposure to DNA damage stimuli (Fig. 3D). Alternatively, BCR-ABL mRNA itself undergoes RNA editing, and results in nucleotide changes at the RNA level (Fig. 3D). Notably, the gene mutation from cDNA was compared, future studies comparing the DNA and RNA differences would aid in confirming the hypothesis.

\section{Discussion}

Resistance to the BCR-ABL inhibitor imatinib poses a major problem for the treatment of CML. IM resistance often results from a secondary mutation in BCR-ABL that interferes with drug binding (2). In the current study, it was identified that mutations of BCR-ABL are selective over the control genes under imatinib treatment. However, within the BCR-ABL gene, mutations may be random. The well-known T315I mutation and others accounting for resistance to multiple TKIs appear to be the results of clones with survival advantages.
In the present study, it was observed that imatinib actively and selectively causes sporadic mutations in the BCR-ABL gene. However, the majority of the mutations of BCR-ABL are not the clinically observed T315I mutation. Notably, certain mutations are silent mutations, which would not confer survival advantages. Therefore, the T315I mutation may be due to clonal expansion of cells with survival advantages. Due to the evolutionary advantage within the tumor environment, the T315I mutation may become dominant in the tumor over time $(12,13)$. As for treatment resistance, surviving drug resistant cells which contain a selective advantage will survive and replicate to repopulate the tumor. In addition, the data of the current study additionally indicated that there are different mutations in the resistant cells, which suggest heterogeneity of drug resistance. It is also important to mention that although imatinib pressure was persistent in the cell model, while clinically-associated T315I and other mutations were not detected, which may be explained by the fact that the clinical selection pressure may be more complicated than solely imatinib or the time differences. To further confirm the clinical relevance of the study, monitoring of the BCR-ABL mutation in patients at different times after imatinib treatment, rather than solely in the final resistant cells, is required. Mutations observed in the present study may be predicted in the early clinical samples immediately after imatinib treatment.

Concerning the mechanism of how imatinib-induced resistance selectively causes BCR-ABL mutation, two hypotheses are proposed. It is reported that DNA double-strand breaks (DSBs) which is suggested to translocate to recurrent DSBs are enriched around the transcription start sites (TSSs) of active genes. This suggests that highly transcribed genes are subject to TSS-associated DSBs which may result in 
Table I. Mutation of BCR-ABL and control gene in parental and imatinib resistant cells.

Mutations of BCR-ABL

Mutated clones of

Cell line

Silent

mutations
Frameshift

mutations
Mutated clones

of control gene in total

\begin{tabular}{llllll}
\hline K562 & $0 / 6$ & 0 & 0 & 0 & $0 / 6$ \\
K562G & $4 / 11^{\mathrm{a}}$ & 2 & 2 & 1 & $0 / 6^{\mathrm{b}}$ \\
$\mathrm{K} 562 \mathrm{R}$ & $6 / 9^{\mathrm{c}}$ & 5 & 4 & 0 & $1 / 10^{\mathrm{d}}$
\end{tabular}

The frameshift mutation occurs in the same clone with a silent mutation at another position. Statistical analysis was completed using the Chi-square test. ${ }^{\mathrm{a} B C R}-\mathrm{ABL}$ in K562G vs. K562, $\mathrm{P}=0.091195$; ${ }^{\mathrm{b}}$ control gene vs. BCR-ABL in K562G, P=0.091195; ' $\mathrm{b}$ : BCR-ABL in K562R vs. K562, $\mathrm{P}=0.009823$; ${ }^{\mathrm{d}}$ control gene vs. $\mathrm{BCR}-\mathrm{ABL}$ in $\mathrm{K} 562 \mathrm{R}, \mathrm{P}=0.010566$.

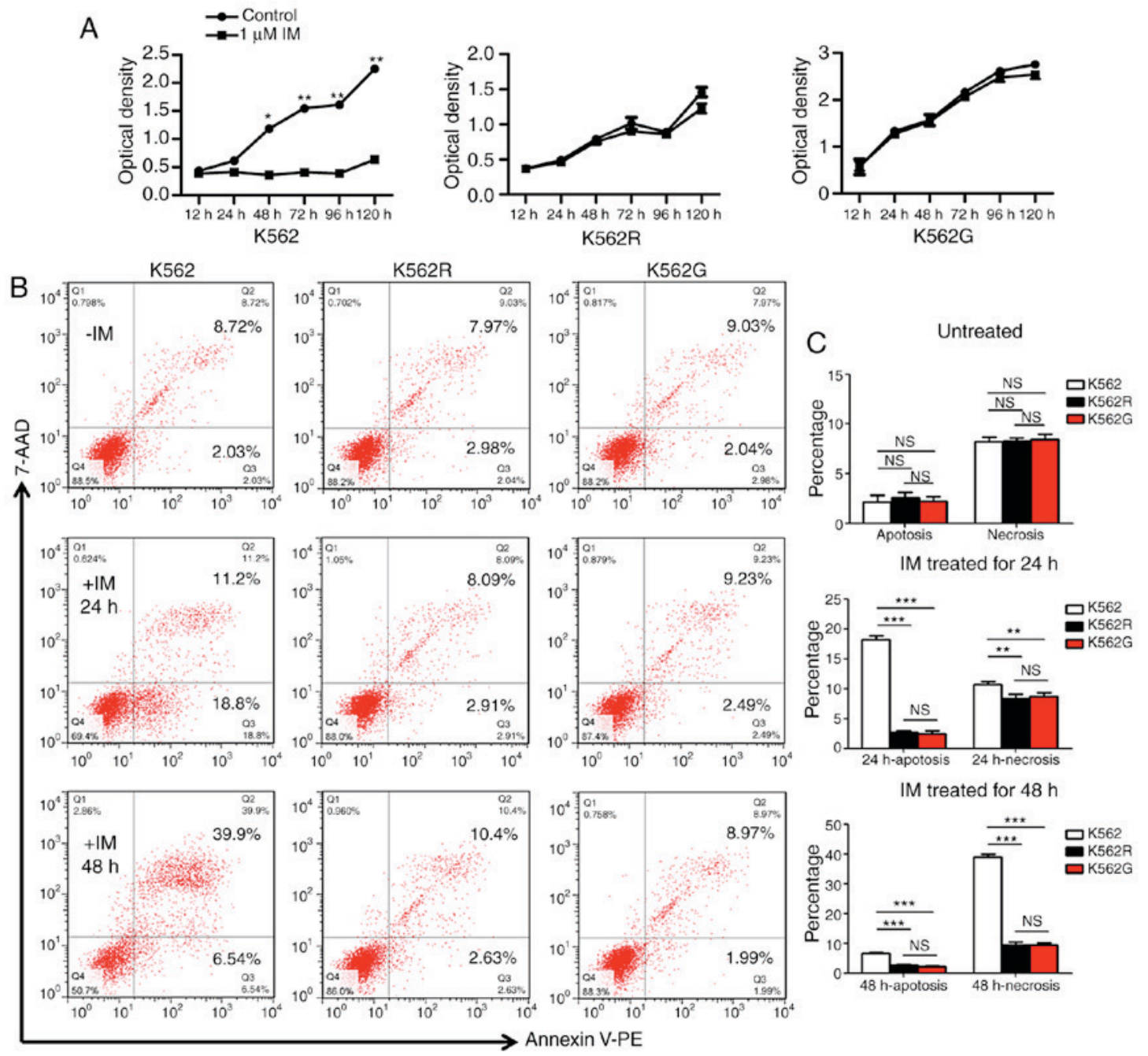

Figure 2. Validation of the imatinib resistance of K562R and K562G cells. (A) K562 cells (left), K562R cell model (middle) and K562G cell model (right) were treated with vehicle or $1 \mu \mathrm{M}$ imatinib for the indicated time periods, and the surviving cells were analyzed by Cell Counting Kit-8. Significant reductions of survival were observed in K562 cells, however not in $\mathrm{K} 562 \mathrm{R}$ and $\mathrm{K} 562 \mathrm{G}$ cells. ${ }^{*} \mathrm{P}<0.05$, ${ }^{* *} \mathrm{P}<0.01$ vs. $1 \mu \mathrm{M}$ imatinib; $\mathrm{n}=5$. (B) K562, K562R and $\mathrm{K} 562 \mathrm{G}$ cells were untreated or treated with $1 \mu \mathrm{M}$ imatinib for 24 and $48 \mathrm{~h}$, followed by analysis of apoptosis using Annexin V/7-AAD staining assay. The apoptosis and necrosis rates of K562 cells with $1 \mu \mathrm{M}$ imatinib treatment were significantly higher than K562R and K562G cells. (C) Quantification data of (B) ${ }^{* *} \mathrm{P}<0.01,{ }^{* * *} \mathrm{P}<0.001$ analyzed by one-way analysis of variance with Tukey's post hoc test; $\mathrm{n}=3$. 7-AAD, 7-amino-actinomycin D; IM, imatinib mesylate; NS, nonsignificant.

translocation in divergent cell types (14). In addition, DSBs repaired through nonhomologous end-joining or homologous recombination may result in various mutations (15). Therefore, it is hypothesized that the altered BCR-ABL activity may be associated with a compensatory increase of BCR-ABL transcription, which is now undergoing. 
A

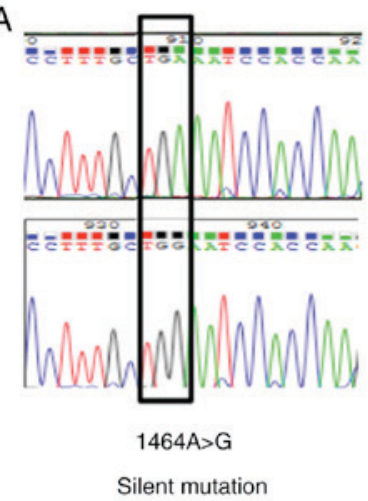

B

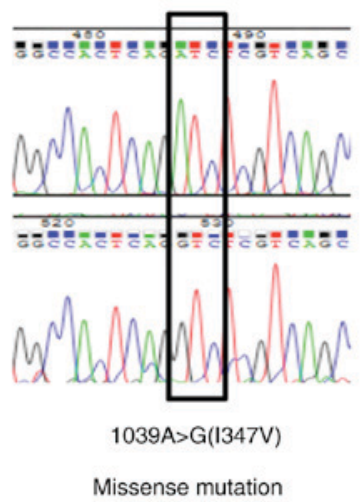

Missense mutation
C
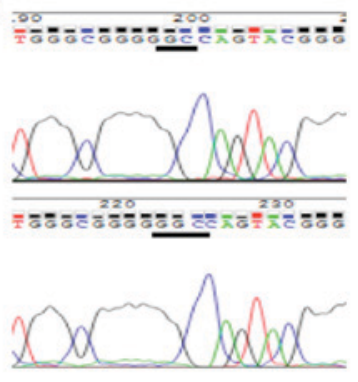

752_753insG

Frameshift mutation

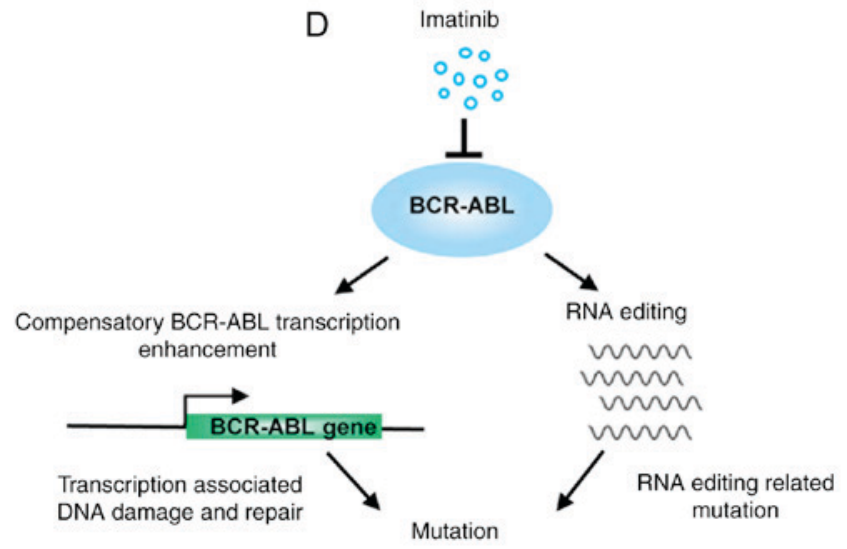

Figure 3. Selective mutation of BCR-ABL gene in imanitib resistant cells and putative hypothesis. (A) Representative sequencing results of silent mutation of BCR-ABL in K562G: Adenine (A) at mRNA position 1464 (in the ABL sequence) was replaced with guanosine (G). (B) Representative sequencing results of missense mutation of BCR-ABL in K562G: A at mRNA position 1039 (in the ABL sequence) was replaced with G, which resulted in isoleucine (I) to valine (V) at position 347. (C) Representative sequencing results of a frameshift mutation of BCR-ABL in K562G: Insertion of G at 752. (D) Proposed hypothesis of selective BCR-ABL mutation in imatinib resistant cells. Inhibition of imatinib treatment to BCR-ABL activity would result in the compensatory transcriptional enhancement of BCR-ABL, which may increase the chance of DNA damage due to exposure of naked DNA to external detrimental stimuli. Alternatively, BCR-ABL mRNA itself undergoes RNA editing, resulting in nucleotide change at RNA level or RNA guided DNA mutation.

It is additionally known that RNA editing increases the diversity of transcriptomes and proteomes in eukaryotic organisms through post-transcriptional modifications of mRNA sequences (16). The forms that RNA editing modifies mRNA sequences include insertion, deletion and the most common type, base substitution (17). RNA editing alters codons to directly modify the coded amino acid or regulate the stability of particular molecules in the introns and untranslated regions $(18,19)$. It is thus also possible that RNA editing may be an important mechanism of BCR-ABL mutation, which is likely, particularly due to the fact that the sample used in the current study was RNA.

Additionally, acquired drug resistance, such as imatinib resistance, develops after an average of 1 year of continuous drug treatment, likely due to the development of secondary mutations of drug target genes, or activation of alternative signaling pathways. For example, an epidermal growth factor receptor (EGFR) secondary mutation in exon 20 (T790M) accounts for approximately $60 \%$ of cases with acquired resistance to EGFR-TKI therapy in non-small-cell lung carcinoma patients (20). Genome instability acts as a fuel for cell-to-cell variation, which ultimately gives rise to selection and evolution (21). In addition, the stability and integrity of the human genome are maintained by the DNA damage repair system. Notably, unrepaired DNA damage serves an essential role in potentially mutagenic lesions that drive carcinogenesis (22). For example, heterozygous germline mutations in breast cancer 1 or 2 (BRCA1/2) are responsible for developing a large fraction of cancer types, and are capable of markedlyincreaseing the lifetime risk of breast cancer (23). In addition, individuals with germline mutations in mismatch-repair genes (primarily mutL homolog 1 and mutS homolog 2) also account for approximately $60 \%$ of cases of hereditary non-polyposis colorectal cancer (24). Therefore, the acquisition of DNA repair gene mutations results in genome instability, which may result in tumor heterogeneity that can contribute to treatment failure and drug resistance. It is thus interesting to test whether genome instability and DNA repair mechanisms are involved in the process.

It is important to note the limitations of the current study. Due to funding limitations, the mutation profile was not analyzed genome wide. In addition, the data here could not confirm the exact level where the mutation originated, which may occur at either a DNA or RNA level or both. Future studies using RNA-sequencing together with exon-sequencing may aid in elucidating this.

In conclusion, it was identified that imatinib actively and selectively causes sporadic mutations in the BCR-ABL gene, while not in the control gene. In addition, mutations within the BCR-ABL appear to be random and it can be inferred that 
there may be different resistant cell types present in different patients. Notably, the majority of the mutations of BCR-ABL are not the clinically observed T315I mutation, suggesting that the clinical selective stress may not be solely imatinib-dependent. Taken together, the results of the present study demonstrated the mutagenesis process during drug resistance and thus may aid in the management of chemotherapy.

\section{Acknowledgements}

The current study was funded by the National Natural Science Foundation of China (grant no. NSFC31572344 to Dr Li Liu).

\section{References}

1. Huang $\mathrm{X}$, Cortes $\mathrm{J}$ and Kantarjian $\mathrm{H}$ : Estimations of the increasing prevalence and plateau prevalence of chronic myeloid leukemia in the era of tyrosine kinase inhibitors therapy. Cancer 118: 3123-3127, 2012.

2. Ma L, Shan Y, Bai R, Xue L, Eide CA, Ou J, Zhu LJ, Hutchinson L, Cerny J, Khoury HJ, et al: A therapeutically targetable mechanism of BCR-ABL-independent imatinib resistance in chronic myeloid leukemia. Sci Transl Med 6: 252ra121, 2014.

3. Hughes TP, Kaeda J, Branford S, Rudzki Z, Hochhaus A, Hensley ML, Gathmann I, Bolton AE, van Hoomissen IC, Goldman JM, et al: Frequency of major molecular responses to imatinib or interferon alfa plus cytarabine in newly diagnosed chronic myeloid leukemia. N Engl J Med 349: 1423-1432, 2003.

4. Berman E, Jhanwar S, Hedvat C, Arcila ME, Wahab OA, Levine R, Maloy M, Ma W and Albitar M: Resistance to imatinib in patients with chronic myelogenous leukemia and the splice variant BCR-ABL1 (35INS). Leuk Res 49: 108-112, 2016.

5. Tabarestani S and Movafagh A: New developments in chronic myeloid leukemia: Implications for therapy. Iran J Cancer Prev 9: e3961, 2016.

6. Wang LX, Wang JD, Chen JJ, Long B, Liu LL, Tu XX, Luo Y, Hu Y, Lin DJ, Lu G, et al: Aurora A kinase inhibitor AKI603 induces cellular senescence in chronic myeloid leukemia cells harboring T315I mutation. Sci Rep 6: 35533, 2016.

7. Bixby D and Talpaz M: Seeking the causes and solutions to imatinib-resistance in chronic myeloid leukemia. Leukemia 25: $7-22,2011$

8. Gupta P, Kathawala RJ, Wei L, Wang F, Wang X, Druker BJ, Fu LW and Chen ZS: PBA2, a novel inhibitor of imatinib-resistant BCR-ABL T315I mutation in chronic myeloid leukemia. Cancer Lett 383: 220-229, 2016.

9. Speijer D: Does constructive neutral evolution play an important role in the origin of cellular complexity? Making sense of the origins and uses of biological complexity. Bioessays 33: 344-349, 2011.
10. Song Y, Shi Y, Carland TM, Lian S, Sasaki T, Schork NJ, Head SR, Kishi S and Schimmel P: p53-Dependent DNA damage response sensitive to editing-defective tRNA synthetase in zebrafish. Proc Natl Acad Sci USA 113: 8460-8465, 2016.

11. Burger G: Non-functional genes repaired at the RNA level. C R Biol 339: 289-295, 2016.

12. Nowell PC: The clonal evolution of tumor cell populations. Science 194: 23-28, 1976.

13. Gerlinger M, Rowan AJ, Horswell S, Math M, Larkin J, Endesfelder D, Gronroos E, Martinez P, Matthews N, Stewart A, et al: Intratumor heterogeneity and branched evolution revealed by multiregion sequencing. N Engl J Med 366: 883-892, 2012.

14. Schwer B, Wei PC, Chang AN, Kao J, Du Z, Meyers RM and Alt FW: Transcription-associated processes cause DNA double-strand breaks and translocations in neural stem/progenitor cells. Proc Natl Acad Sci USA 113: 2258-2263, 2016.

15. Ceccaldi R, Rondinelli B and D'Andrea AD: Repair pathway choices and consequences at the double-strand break. Trends Cell Biol 26: 52-64, 2016.

16. Li JB and Church GM: Deciphering the functions and regulation of brain-enriched A-to-I RNA editing. Nat Neurosci 16: 1518-1522, 2013.

17. Witzany G: The agents of natural genome editing. J Mol Cell Biol 3: 181-189, 2011.

18. Grewe F, Viehoever P, Weisshaar B and Knoop V: A trans-splicing group I intron and tRNA-hyperediting in the mitochondrial genome of the lycophyte Isoetes engelmannii. Nucleic Acids Res 37: 5093-5104, 2009.

19. Castandet B, Choury D, Bégu D, Jordana X and Araya A: Intron RNA editing is essential for splicing in plant mitochondria. Nucleic Acids Res 38: 7112-7121, 2010.

20. Zhang X, Maity T, Kashyap MK, Bansal M, Venugopalan A, Singh S, Awasthi S, Marimuthu A, Charles Jacob HK, Belkina N, et al: Quantitative tyrosine phosphoproteomics of Epidermal Growth Factor Receptor (EGFR) tyrosine kinase inhibitor-treated lung adenocarcinoma cells reveals potential novel biomarkers of therapeutic response. Mol Cell Proteomics 16: 891-910, 2017.

21. McGranahan N and Swanton C: Clonal heterogeneity and tumor evolution: Past, present, and the future. Cell 168: 613-628, 2017.

22. Gao D, Herman JG and Guo M: The clinical value of aberrant epigenetic changes of DNA damage repair genes in human cancer. Oncotarget 7: 37331-37346, 2016.

23. King MC, Marks JH and Mandell JB; New York Breast Cancer Study Group: Breast and ovarian cancer risks due to inherited mutations in BRCA1 and BRCA2. Science 302: 643-646, 2003.

24. Yu H, Li H, Cui Y, Xiao W, Dai G, Huang J and Wang C: The mRNA level of MLH1 in peripheral blood is a biomarker for the diagnosis of hereditary nonpolyposis colorectal cancer. Am J Cancer Res 6: 1135-1140, 2016. 